\title{
Vivir la danza contemporánea desde América Latina. Un nuevo mundo que conquistar. Historia de vida
}

Living Contemporary Dance from Latin America. A New World to Conquer. A Life Story

\section{Viva a dança contemporânea da América Latina. Um mundo novo a conquistar. Historia de vida}

Santiago López-Corrales

Economista

Bailarín y coreógrafo, grupo Humanizados

Centro de Estudios Generales

Universidad Nacional de Costa Rica

Recibido: 5/6/2017 Aprobado: 15/10/2017

\begin{abstract}
Recuerdos que no se olvidan, experiencias, fantasías que cambian la vida, un pueblo lejano con muchas travesías y mucho que contar...

llegó la danza a mi vida, llena de esperanza e ilusión, con diversas oportunidades, un nuevo mundo que solo en sueños podía imaginar. Recuerdos que no se olvidan, experiencias, fantasías que cambian la vida, un pueblo lejano con muchas travesías y mucho que contar...

llegó la danza a mi vida, llena de esperanza e ilusión, con diversas oportunidades, un nuevo mundo que solo en sueños podía imaginar
\end{abstract}

Desde pequeño bailaba. Bueno en ese entonces no le podía decir baile porque era pecado, más bien le decíamos danza y solo lo podía hacer dentro de la iglesia para alabar y adorar a Dios, al menos eso fue lo que

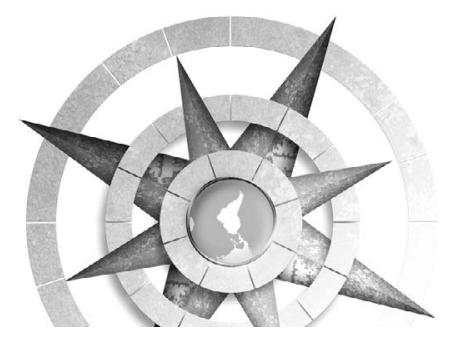

DOI: http://dx.doi.org/10.15359/tdna.33-62.6 aprendí desde los ocho años, ahí empecé mis primeros pasos en la danza, en coreografías que los guías nos enseñaban en la escuelita dominical los domingos.

Fue hasta los diecisiete años ya en quinto año de escuela secundaria (me faltaba un año para salir, en el Colegio Técnico Profesional de Quepos distrito de la provincia de Puntarenas, mis tres primeros años fueron en el CTPA de Matapalo, 
Pacífico Sur de Costa Rica) a finales de este año un amigo cercano me habló de un grupo de danza y me dijo que él ya tenía un año de ser parte del mismo, me invito a uno de los ensayos, el grupo se llamaba Saragundí (actualmente Corpóreos), no le tomé mucha importancia, hasta que un día me decidí a ir, cuando llegué y vi lo que estaban haciendo, un señor pelón con una barba negra los entrenaba (era el maestro coreógrafo Carlos Ovares), mis ojos hasta que brillaron de ver lo que sucedía en el escenario, yo quería estar ahí, lo que hacían los chicos ya lo había vivido (en mis sueños), no tenía palabras, apenas terminó el ensayo, corrí, hablé con el coreógrafo, le dije que quería entrar al grupo y le pregunté cuáles eran las condiciones para ser parte del mismo.

Todo inició en ese momento, una experiencia maravillosa, entrenábamos tres veces por semana, de $6 \mathrm{p}$. m. a 9 p. m., dedicábamos entre una y dos horas de entrenamiento, y una hora de montaje coreográfico, cada montaje tardaba alrededor de seis meses y otros seis de giras por todo el país, en

Figura 1. "Compañía Bananera”, 2009, ASOMUFACQ Coreógrafo: Carlos Ovares

Fuente: archivo fotográfico del autor.

escuelas y colegios; Carlos Ovares era el coreógrafo del grupo, él nos motivó a bailar, llevó danza hasta nuestro pueblo con ayuda de la Asociación Mujer, Familia y Comunidad de Quepos (ASOMUFACQ), organización encargada de la parte administrativa y la generadora de fondos para vestuario, transporte, hospedaje y alimentación en las giras, su representante y se podría decir que directora del grupo era Julia Vargas Molina.

Fui parte de esta agrupación por dos años, en el primer año entré sin saber nada de la danza, además estaba en el último año de colegio y me preparaba para exámenes de bachillerato, salía de clases directo al ensayo, como dije tres veces por semana, y 


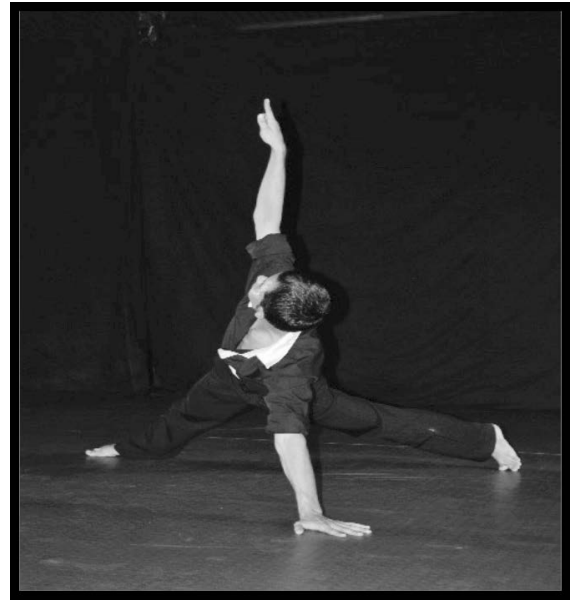

Figura 2. "Hay lobos con piel de cordero", 2009

Coreógrafo: Carlos Ovares

Fuente: archivo fotográfico del autor.

a esto hay que sumarle las giras que se realizaban por lo general durante los fines de semana. Participamos por primera vez en el Festival de Coreógrafos Graciela Moreno 2009 y luego en el 2010, gracias a la iniciativa del Mtro. Carlos Ovares por incentivar la participación de un grupo aficionado en un festival tan reconocido en el nivel nacional, claro que hubo polémica alrededor de todo esto; sin embargo para nosotros fue un experiencia llena de adrenalina, sentimos cómo por primera vez pisamos las tablas de un teatro, y el más reconocido en todo el país como lo es el Teatro Nacional; a pesar que ya habíamos bailado en diferentes lugares, estar en el teatro no tenía explicación.

Me gustaría mencionar las obras coreográficas que tuve el honor de bailar en el grupo de Danza Saragundí: mi primera participación fue en la obra que representó a la compañía bananera, United Fruit Company y todo el proceso de colonización en la zona de Quepos; la segunda se llamó La purísima, era la representación de las festividades religiosas y todo el ritual que le acompaña; la tercera coreografía se tituló Hay lobos con piel de cordero, que trata sobre el abuso sexual y la violencia, se estrenó en el Festival de Coreógrafos 2009; la cuarta y última coreografía que bailé en dicha agrupación Juegos violentos también se estrenó en

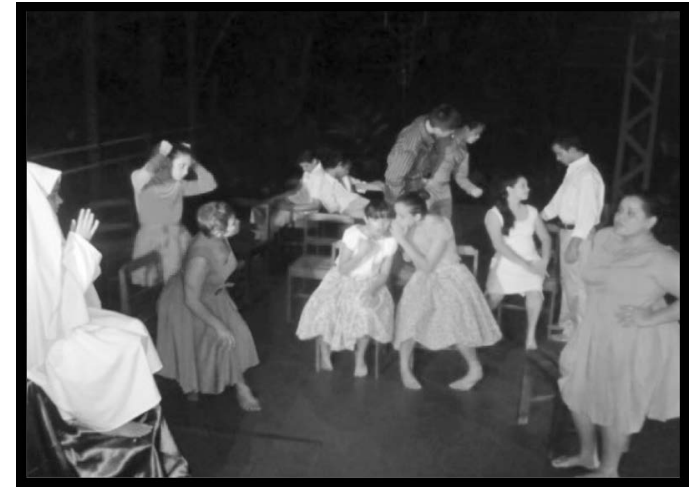

Figura 3. "La purísima", 2009 Coreógrafo: Carlos Ovares

Fuente: archivo fotográfico del autor. 
el Teatro Nacional en el Festival de Coreógrafos 2010.

El 2010 fue un periodo de decisión y de ahorro porque mis intenciones eran irme a estudiar a la capital, mi prioridad era entrar a la Universidad de Costa Rica (UCR) en San Pedro, ya sabía que quería estudiar Economía, creía que la mejor universidad pública era la UCR, realicé en el 2009 el examen de admisión de dicha institución, la nota que obtuve no me dio para aplicar en la carrera de Economía, por lo que decidí hacer nuevamente el examen de ingreso para la Universidad de Costa Rica (UCR) y adicionalmente realicé el de la Universidad Nacional (UNA), fui seleccionado para entrar directo a la carrera de Economía en la UNA en el 2011.

Iniciando en el 2011 un nuevo periodo de mi vida en la Universidad Nacional y dejando atrás tantas cosas (danza, amor, trabajo, familia, amigos...). El primer lugar donde viví fue en el Roble de Alajuela, en casa de un tío y aprendí a sobrevivir con los ahorros que hice durante todo un año, que no era mucho.

Estaba tan decidido a ser economista, pero lo que había dejado en Quepos no estaba en Heredia. Tenía que empezar de cero, fue cuando debatí si realmente quería seguir bailando, añoraba la danza, a pesar de que no dejaría mi carrera en Economía abandonada, sí quería bailar. La Universidad no me ofrecía mucho, solo habían dos grupos de baile para estudiantes regulares, en los grupos representativos universitarios, como Barbac, de baile folclórico, y Querube, de baile popular. ¿Dónde está la danza contemporánea? Solo en la Escuela de Danza, como carrera. Esa era la única opción que estaba en ese momento y, por un semestre, era el curso de Movimiento creativo impartido por el profesor Carlos Morúa Carrillo, en el Centro de Estudios Generales (CEG). Barbac es un grupo de danza folclórica y Querube es de bailes populares, pero mi pasión es la danza contemporánea y explorar el movimiento desde otros estilos de baile, esto solo en el curso de Movimiento creativo podía hacerlo.

Claro que aproveché al máximo el curso, pero lo bueno no dura para siempre, gracias a que conocí al profesor Morúa, me incentivó a realizar la prueba para ingresar a la Escuela de Danza, estaba con un deseo enorme de llevar las dos carreras, la de Danza y la de Economía, luego de hacer los trámites respectivos, y hacer la prueba específica de carrera, llegué a quedar elegible para entrar a Danza, solo había que esperar los

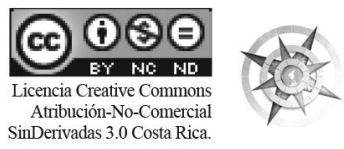


resultados, sumar la nota de admisión y la nota obtenida en la prueba, y listo, a cumplir mi sueño. Pero no fue así. Me llamaron de la Escuela de Danza y me dieron la mala noticia -le informamos que no puede ser elegido por que usted estudia otra carrera y el requisito para optar por una segunda carrera es que debe tener un mínimo de 30 créditos aprobados- o sea, debía terminar el primer año de carrera, realizar la prueba nuevamente, en el segundo año de economía (2012), y pasar otra vez el mismo proceso de selección para ingresar en el tercer año de mi primer carrera (2013), me sentí angustiado, decepcionado de la vida, de la Universidad, de la poco información que recibí del Departamento de Registro en ese entonces, y para terminar de agravar la situación, perdí un curso en el primer semestre (Matemática 1 para Economía). ¡Peor! No servía para la Economía (en vez de economía) y no servía para la economía y no podía estudiar Danza, eso fue lo que pensé en ese momento.

El profesor Carlos Morúa me dio la oportunidad que fuera a sus clases de Movimiento creativo en el segundo semestre (2011) como asistente, aún había esperanzas de bailar y entrenarme, el me dio la oportunidad de montar mi primer trabajo coreográfico "Alcanzar el vuelo" con estudiantes del curso, fue una gran oportunidad, en resumen, la obra trató de la lucha por los sueño y no rendirse nunca... Si caemos, levantarnos otra vez e iniciar de nuevo, alcanzar el vuelo... (Alcanzar el vuelo, 2011). Este trabajo coreográfico de alguna manera me permitió descargar todas esas emociones negativas como la soledad, la decepción, el engaño y las emociones positivas como la esperanza, la fe, la superación, todo lo que sentía en ese momento de mi vida, donde me cuestionaba que hacía realmente aquí en la Universidad.

Para finales del 2011, tenía muchas dudas pero estaba decidido a continuar con la carrera de Economía, en la Danza era muy incierto, hasta que recibí una llamada del profesor Morúa donde me invitaba a formar parte de un grupo que se estaba formando en el CEG, claro que acepté, éramos alrededor de once personas, fue como una luz que iluminó mi vida, y me di cuenta que no todo estaba perdido, mi camino en el baile aún continuaba, por cierto tenía 20 años cuando ingrese a la Universidad, me sentía un poco "viejo" para estudiar danza, este estereotipo me limitó a realizar nuevamente la prueba. En la Danza siempre existe el tema de la edad y las condiciones físicas de la persona. Con el tiempo

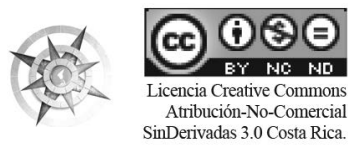

Vivir la danza contemporánea desde América Latina. 111

Un nuevo mundo que conquistar. Historia de vida Santiago López-Corrales 
entendí que no hay límites para bailar, siempre y cuando uno lo desee.

Cuando se formó el grupo Cuerpo colectivo, (fue el primer nombre que se le dio actualmente se denomina Humanizados) mi mente y corazón estaban de lleno en este grupo y mi interés por estudiar danza se fue apagando o más bien, llenando con el grupo de Danza del CEG, considerando que éramos un grupo informal, que no tenía un reconocimiento institucional, los espacios de entrenamiento eran diversos, aulas, pasillos de la Universidad, zonas verdes, etc. Se podría decir que las condiciones eran precarias, sin un lugar fijo para entrenar, mucho menos disponíamos de un ingreso para comprar vestuario o algún reconocimiento por parte de la Universidad por ser parte de un grupo que la representaba. Todos estábamos realmente comprometidos con el grupo y lo que nos motivaba para ir a los ensayos era el deseo de bailar, distraernos de lo cotidiano, de sentirnos en familia.

En el 2012 se creó la obra Una hoja de aire fue la primera puesta en escena del grupo Humanizados, dirigida por David Rojas Lobo, donde participamos los primeros integrantes del grupo (Alejandra Montero, Heidy González, Juan Ruíz, Luis Ruiz, Juan Gabriel Oviedo, Karen Ruiz, Raquel
Sánchez, Xiomara Naranjo y Santiago López), para la mayoría fue un reto, algunos apenas iniciaban sus primeros pasos en el baile y por otro lado teníamos que aprender técnicas de teatro, la puesta en escena era como un experimento entre teatro y danza, ensayábamos tres veces por semana, en los pasillos de Generales, en aulas, incluso tuvimos la oportunidad de trabajar en la escuela de Artes Escénicas, había que buscar todas las posibilidades, no éramos un grupo oficial de la Universidad, pero teníamos que demostrar que realmente queríamos esto, fue difícil en este periodo de iniciación, algunas veces ensayamos hasta las diez de la noche, el esfuerzo, la responsabilidad de parte de todos, el deseo de hacer un buen trabajo y la disciplina, permitió que el grupo se acoplara y creciera profesionalmente.

No todos estábamos contentos con este trabajo, porque queríamos bailar, no hacer teatro, no queríamos hablar en público, realmente fue un proceso algo incómodo, que logramos sacar adelante, aunque algunas veces discrepamos con el director de la obra, nos mantuvimos firmes con la convicción de que el resultado final sería más satisfactorio.

La obra Una hoja de aire fue una adaptación de la novela del mismo 
nombre del escritor Joaquín Gutiérrez, relata la historia de Alfonso, un joven que viaja a México porque quiere ser actor; su sueño nace en la infancia cuando el amor de su vida, Teresa, le regala una hoja de aire a la que le crecen otras hojas, y otras cada vez más pequeña hasta que se las lleva el viento. Esta metáfora representa nuestro mundo lleno de ilusiones, nos dimos cuenta que cada uno tenía sus propios sueños que aún no se cumplían, aspecto que fue quedando plasmado en este trabajo escénico.

Paralelo a esta obra, el director del grupo, Carlos Morúa, realizó un remontaje de un dueto con el nombre de Guerreros, que hasta el día de hoy ha sido emblemática en el grupo, ambas se estrenaron el mismo año que se fundó el grupo (2012); además tuve la oportunidad de remontar mi primer trabajo coreográfico Alcanzar el vuelo, el cual lo venía madurando desde el 2011 cuando el profesor Carlos Morúa, me dio la oportunidad de realizar un montaje con los estudiantes del curso "Taller de Movimiento creativo"; para entonces ya el grupo estaba más sólido, durante todo el semestre realicé el montaje con ayuda de todos y todas las integrantes del grupo, experimentando cualidades propias de movimiento de cada participante.
Recuerdo que en este periodo se dio la primera crisis existencial del grupo, después de la primera temporada de Humanizados, con "Una hoja de aire" y "Guerreros", el grupo se desintegró, éramos nueve en total y quedamos seis, uno de los integrantes era el personaje principal de la obra "Una hoja de aire", después de tanto esfuerzo solo presentamos una vez este montaje, lo que desmotivó bastante al director del grupo. Los que quedamos nos desmotivamos un poco también, pero entendíamos que todo cambia y cuando uno está en la Universidad la vida no es fácil, sin embargo no estábamos

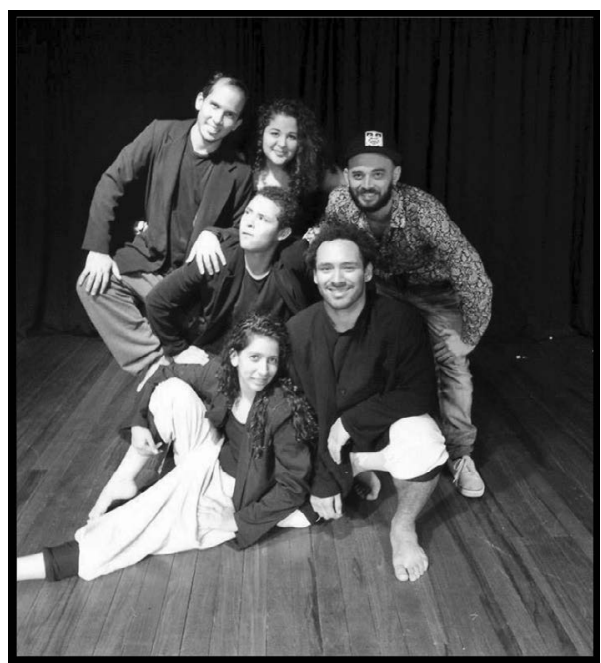

Figura 4. "Eco de Ángeles", Christopher Núñez.

Bailarines: Alejandra Montero, Luis Ruiz, Juan Ruiz y Santiago López.

Fuente: archivo fotográfico del autor. 
dispuestos a tirar la toalla, ese año trabajamos dos veces por semana por nuestra cuenta, siempre haciendo uso del espacio que logramos en el $\mathrm{CEG}$, gracias a la presentación que hicimos con la Hoja de aire, donde se demostró que un grupo de estudiantes de diferentes carreras podían formar una agrupación de la nada y sin ningún recurso y sobretodo, haciendo un trabajo profesional.

En el 2013 éramos cinco integrantes más el director, fue una año diferente, el profesor Carlos Morúa invito a Christopher Núñez un exintegrante de "Danza Alfa", además graduado de la Escuela de Danza de la UNA.

Christopher trabajó con el grupo durante todo ese año con un fuerte entrenamiento físico, era la persona que necesitábamos para unificar al grupo técnicamente y subir de nivel, además que en el primer semestre del mismo año realizó el montaje "Eco de ángeles", esta coreografía implicaba una mayor técnica (en giros, saltos, alzados...), el aporte de este coreógrafo al grupo fue en grandes proporciones, y no solo como bailarines, también llegó a tocarnos de manera personal. Desde mi punto de vista Christopher hizo que creyéramos más en nosotros mismos, nos impulsaba constantemente a ser mejores personas y bailarines.
En este año se realizó la temporada de Humanizados "Vivencias", donde se estrenó: Alcanzar el vuelo (Santiago López), Gravitación (coreógrafo Juan Ruiz), y Eco de ángeles. Algunas de las personas que integraron el grupo durante este período por diferentes razones salieron: Raquel González, Yeimi Vega, María Fernanda Oses, Adriana Víquez; de manera que para finales del 2013 quedamos únicamente cuatro integrantes: Alejandra Montero, Luis Ruiz, Juan Ruiz y Santiago López. Una de las exintegrantes del grupo Adriana Víquez logró entrar a la Escuela de Danza en el 2014, es una persona con un alma sensible y un deseo enorme de bailar y crecer profesionalmente, tenía una meta clara que logró alcanzar.

Para el 2014 el grupo dio un giro total, se mostró ya consolidado con cuatro bailarines, después de tres años de luchas por un espacio fijo de entrenamiento en CEG y de unificar al grupo técnicamente, lo cual logró con persistencia, entrega, y con la ayuda de un gran guía y director como lo ha sido el profesor Carlos Morúa. Recuerdo como un año entero cada uno ponía de su bolsillo para pagar las clases del coreógrafo Christopher Núñez, para poder recibir clases profesionales de danza contemporánea, porque 


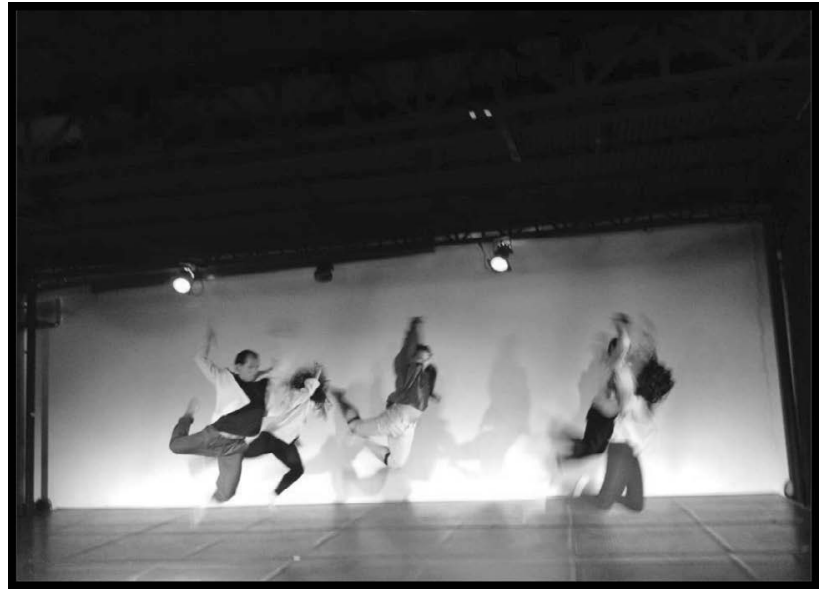

Figura 5. "Alcanzar el vuelo", Santiago López Bailarines: Alejandra Montero, Heidy González, Luis Ruiz, Juan Ruíz y Santiago López.

Fuente: Fotografía gráfica Génesis, 2013 con ayuda del profesor Carlos Morua, un día entrenabamos en la pecera y otro en el salón de Exdecanos.

En el 2014 decidimos crecer y aumentar el número de bailarines, se realizó la primera audición abierta, más que una audición, se les permitió a los interesados participar en una de las clases del grupo y el único factor limitante para ser parte del grupo aunque empezamos a conquistar un espacio en el CEG, el salón de Exdecanos no tenía las condiciones idóneas para entrenar, no había espejos ni barras y el piso no era de madera, cualquier persona que esté en el medio sabe que estos recursos son necesarios para lograr avanzar técnicamente y proteger al bailarín de una lesión, ya que el trabajo en condiciones no aptas como el piso de cerámica, puede causar lesiones en las rodillas al momento de saltar; con todo esto, igual fue un año de gran avance para el grupo.

Por otro lado, entrenamos con Christopher en su estudio "La pecera" por nuestros propios medios y eran ellos mismos, siempre nos consideramos un grupo abierto, donde no existe ninguna discriminación, la idea era diversificarnos en estilos de baile y disfrutar haciéndolo.

Gracias a esta audición se incorporaron un grupo de chicas muy valiosas, ocho en total: Laura Navarro, Yoselyn Campos, Carolina Ruiz, Vicky Angoca, Noelia, Kaysí Gómez, Maria José Ortiz y Gabriela Obando; y tres hombres: Uriel Morera, Jasón Cerdas, y Ricardo Prezord. Tuve la oportunidad de hacer un segundo montaje coreográfico "Soy mujer" con los integrantes nuevos del grupo, en su mayoría mujeres, por lo que me inspire en el Día Internacional de la 


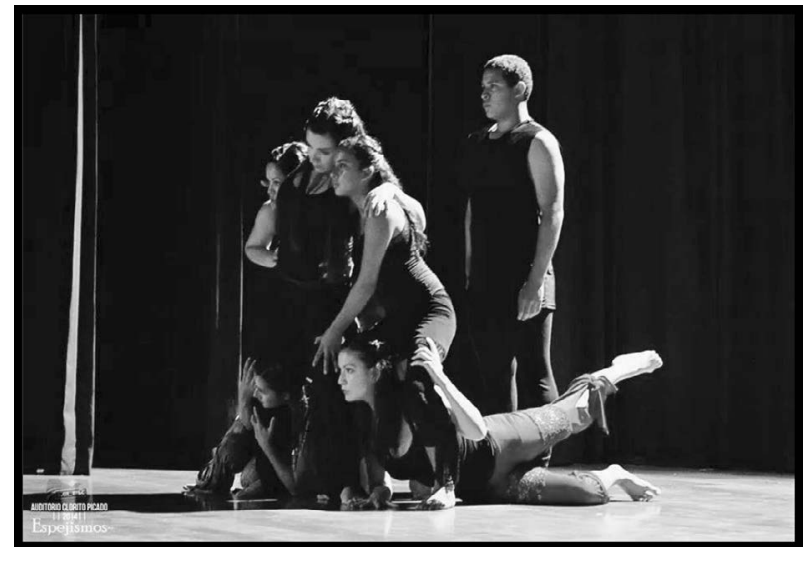

Figura 6. "Soy Mujer", Santiago López.

Bailarines: Laura Navarro, María Ortiz, Gabriela Obando,

Carolina Ruíz, Noelia, Kaysí Gómez y Luis Ruiz. Auditorio Clodomiro Picado, 2014

Fuente: archivo fotográfico del autor.

implicaba un reto más grande y el entrenamiento iba más allá de la técnica, se necesitaba elasticidad y resistencia; su coreografía era de constante movimiento, saltos, alzadas, correr durante 15 minutos, el tiempo que tardaba esa coreografía no era lo que nos preocupaba, ya que era muy desgastante y nosotros cuatro bailábamos casi todas las coreografías, sobre

Mujer. Fue un trabajo muy personal donde cada chica debía investigar sobre una mujer que para ellas representara un ejemplo a seguir y luego plasmar esa lucha personal de la mujer por la igualdad y la equidad, en movimiento y expresión corporal.

En este mismo año se trabajó en nuevos montajes coreográficos para el grupo, tuvimos como coreógrafa invitada a Lourdes Cubero (Lula) profesora del Castella, graduada de la Escuela de Danza y fue parte del grupo Danza Alfa, ella montó una coreografía con el nombre de "Kuántico", donde bailaron: Luis Ruiz, Alejandra Montero, Juan Ruiz y Santiago López. Este montaje todo cuando los espectáculos que realizábamos eran de una hora, solo hay que imaginar lo que es estar una hora bailando sin parar, y una vez que el espectáculo terminaba, caíamos todos muertos.

El resultado final de este montaje fue muy interesante y bastante abstracto, lo que costaba para el público comprender la puesta en escena; para nosotros como bailarines fue crecer profesionalmente, estábamos muy agradecidos por trabajar con Lula, un ser humano alegre, y aunque pequeña es una mujer explosiva, en el buen sentido de la palabra, siempre logrando lo que se propone con su carisma y personalidad. 
En el segundo semestre de ese mismo año se realizó un montaje Teatro-Danza con la colaboración del Mtro. Rolando Salas, director del grupo de teatro Unánime, "Esta te la dedico". A grandes rasgos trataba de las rupturas emocionales que vivimos en la vida. Una vez más sentimos que el trabajo era otro experimento, la mezcla entre lo corporal y oral, este proceso permitió acoplar a los integrantes nuevos del grupo, también fue un momento personal donde cada uno exploró en su interior y si había alguna herida emocional que no había sanado, era la oportunidad perfecta para sacarlo y seguir adelante, por lo que este montaje fue más allá de lo que podíamos percibir a simple vista.

Se unieron otras bailarinas al grupo poco después de las audiciones, Kristina Quirós y doña Delia Castañeira que es una estudiante del Programa de Atención Integral de la Persona Adulta Mayor (PAIPAM), realizo algunos montajes en el grupo "Son cubano" y "Mambo", además es una bailarina espectacular, tiene un solo que presenta a menudo cuando el grupo realiza funciones "Rumbera", bailó además con el grupo Humanizados en la coreografía "Gente de cuerpo y raíz", montaje que se realizó en el 2015 con la dirección del reconocido maestro Claudio Taylor, uno de los padrinos del grupo, que siempre se ha hecho presente dándonos todo su apoyo de diferentes maneras. Kristina por otro lado era estudiante de Christopher Núñez, y formó parte del grupo alrededor de un año, entró a la Escuela de Danza en el 2015, donde está cumpliendo sus sueños.

El 2015 fue un año de muchos cambios (último año de carrera, la realización de la práctica profesional (PPS), I Encuentro Artístico Humanista), estaba lleno de cosas y con el tiempo muy reducido, en abril justo después de Semana Santa empecé la Práctica Profesional Supervisada (PPS) en mi carrera de Economía, la cual realice en la Cuenta Satélite de Cultura del Ministerio de Cultura y Juventud(MCJ), justo cuando llevaba los cursos más difíciles "Econometría”, y se venía el "I Encuentro Internacional Artístico Humanista" (I EIAH), tenía que sacar tiempo para apoyar al grupo, durante el día de 8 a.m. a 3 p.m. estaba en la PPS y luego regresaba a Heredia a recibir clases de 5 p.m. a 9 p.m., eso era todos los días, si acaso solo tenía un par de horas para entrenar en el grupo (4-6 p.m.), lo que afectó mi condición física e impidió que participara de lleno en el nuevo montaje, que inició justo este año con el maestro Claudio Taylor "Gente de cuerpo y

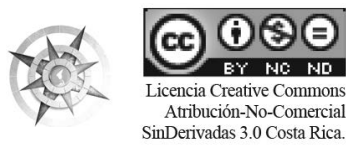

Vivir la danza contemporánea desde América Latina. 117

Un nuevo mundo que conquistar. Historia de vida Santiago López-Corrales 


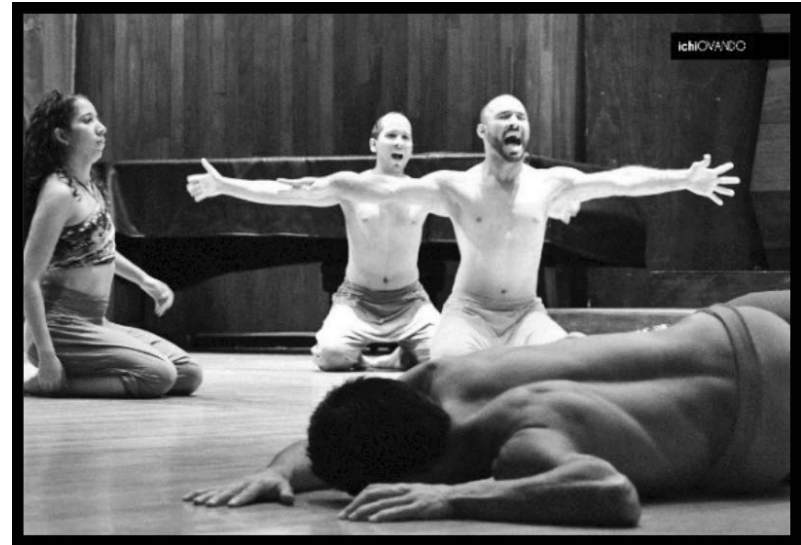

Figura 7. "Gente de cuerpo y raíz", Claudio Taylor Fuente: Fotografía FAM, México, 2016

él siempre tuvo fe en nosotros y en el encuentro, la verdad el encuentro dio mucho de qué hablar de manera positiva, tanto así que se hablaba del "II EIAH 2016".

Ese año el profesor me pidió que presentara la coreografía "Soy mujer" en el Danzaton 2015, era la primera vez que raíz", baile africano, que rescata la cultura limonense. Claudio se ha hecho presente en el grupo desde que iniciamos, nos ha brindado su apoyo emocional, una persona muy reconocida y respetada en el nivel nacional por su trabajo en la danza afro-caribeña.

Se acercó el "I EIAH", el cual implicaba mucha dedicación, atender a las agrupaciones nacionales y extranjeras invitadas. Era toda una experiencia nueva para el grupo, pero creo que nos permitió crecer aún más, desarrollar una actividad como esta en poco tiempo, con el mínimo de presupuesto, y además con solo los integrantes del grupo, era algo que ni yo mismo sabía como lo íbamos a resolver, y lo que más me motivó fue el profesor Carlos Morúa porque participábamos por lo que me sentí muy honrado de hacerlo, fue todo un éxito, después de tanta presión y limitación del tiempo, cuando uno tiene más cosas que hacer el cuerpo se prepara por sí solo, y la mente funciona de maneras que uno no puede explicar.

Al finalizar el 2015 tuvimos nuevos integrantes: Celeste Montero, Dani Valerio, Bryan Gómez, Roland Porras, Gerald Hidalgo. También se realizó una gira a Nicaragua con todo el elenco, los cuatro más viejos de estar en el grupo ya habíamos hecho una gira a Tola, Rivas de Nicaragua, invitados por el maestro Roberto Picado, quien había estado en Costa Rica en una ocasión para dar un taller y realizar un montaje coreográfico con el grupo, un baile 
que se mantiene activo a la fecha "Talento de TV".

La primera vez que fuimos a Nicaragua tuvimos la dicha de compartir con el pueblo de Tola nuestra pasión, hicimos un pequeño taller con los niños y bailamos con el grupo del maestro Roberto Picado, todo fue una experiencia nueva, desde la rica comida hasta la amabilidad y el trato de las personas, sus bailes folclóricos tradicionales eran hermosos, llamativos y elegantes, teníamos envidia y de la buena, nosotros si acaso podíamos costear los pases del bus que nos llevó hasta Nicaragua, sin embargo había otros factores que no eran de envidiar, como las condiciones económicas y el entorno familiar en que crecen muchos de estos niños que no es el mejor, este viaje fue para el 2014. La segunda vez que pudimos ir en el 2015, casi todo el elenco fue, la Universidad Nacional nos facilitó una buseta, realizamos presentaciones en distintos lugares de Nicaragua y en la Academia Nicaragüense de la Danza, todo gracias a Roberto

Picado que nos invitó nuevamente a su bello país.

La siguiente gira que realizó el grupo fue a Quepos, de regreso a mi tierra natal, compartimos escenario con "Corpóreos" y la Academia Nicaragüense de la Danza. Fue una gran experiencia y muchos sentimientos encontrados, las coreografías presentadas fueron: "Gente de cuerpo y raíz" y "Eco de ángeles"

También tuve la oportunidad de participar en otros trabajos coreográficos, uno de ellos que aún se mantiene activo es un Tango Argentino,

Vivir la danza contemporánea desde América Latina. 119 Un nuevo mundo que conquistar. Historia de vida Santiago López-Corrales 


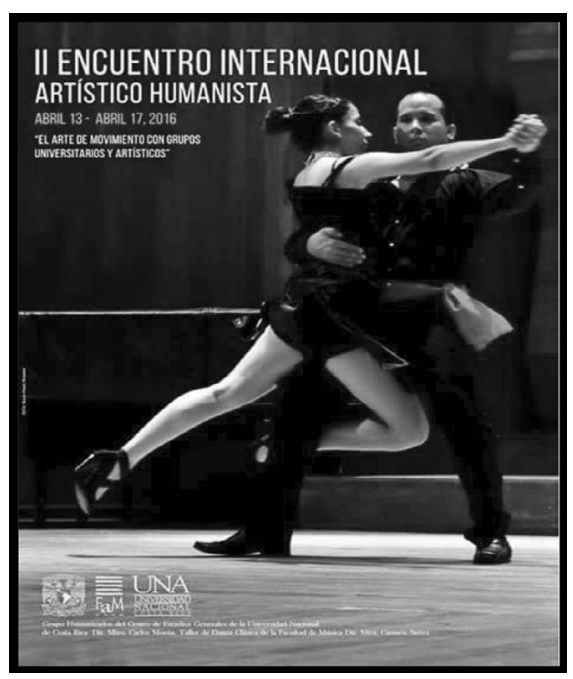

Figura 9. "Tango", Yenier Jiménez

Fuente: Fotografía FAM, México, 2016

dirigido por Yenier Jiménez, se estrenó en el segundo semestre del 2015.

Iniciando el 2016 con mucha adrenalina, Ale (Alejandra Montero) y yo decidimos participar en "Revés 2016", es una plataforma de entrenamiento físico que reúne diferentes tendencias del movimiento durante cuatro semanas, nosotros representamos a Humanizados durante dos semanas. Al mismo tiempo teníamos la gira programada a la Ciudad de México, Universidad Nacional Autónoma de México (UNAM), invitados por la Facultad de Música (FAM), quien hizo posible este encuentro fue la maestra Carmen Sierra (Quirina Orozco), ahí compartimos bellos momentos con sus estudiantes y las excelentes clases magistrales de la maestra y otros invitados.

México nos cambió la vida, esos sueños que teníamos cuando apenas se formaba Humanizados se iban realizando poco a poco, estábamos en México, como decía un gran profesor de Economía, "La realidad es otra" (Hernán Alvarado). No solo fue el viaje a México, eran muchas cosas que pasaron al mismo tiempo, estaba tan feliz, se acercaba el día de mi graduación, organizábamos el "II Encuentro Internacional Artístico Humanista (EIAH) 2016", y también nos preparábamos para participar en el "Danzaton 2016", en el Teatro de la Danza.

Fue un año muy movido, todo pasaba rápido, primero el viaje a México y como les conté fue un sueño hecho realidad, segundo, mi graduación, por lo que había estado luchando tanto tiempo, muchos sacrificios, cinco años lejos de mi familia, sobreviviendo con la beca de la Universidad, haciendo horas colaboración, horas asistente para cubrir los gastos, y tantas desveladas para estudiar, verdaderamente fue una lucha constante, lo único que me hacía salir de la rutina era ser parte del grupo $\mathrm{Hu}$ manizados, donde más que bailar, tenía amigos, una segunda familia, y lo

120 Vivir la danza contemporánea desde América Latina. Un nuevo mundo que conquistar. Historia de vida Santiago López-Corrales

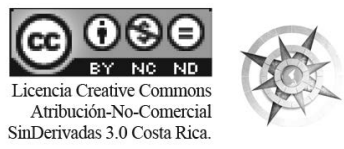


que nos unía, el deseo de expresarnos por medio del movimiento.

Lo tercero fue el "II EIAH 2016", y por último, la presentación en el "Danzaton 2016", participamos con dos propuestas coreográficas: "Guerreros" y "Ya no somos" de Alexandra Delgado. Debo añadir que en este año se in-

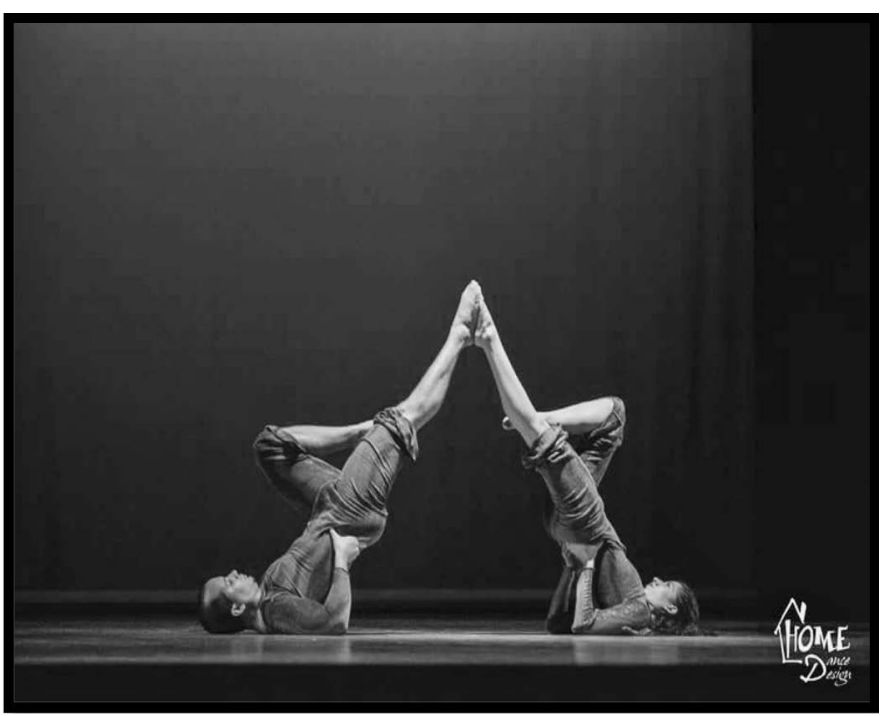

Figura 10. Coreografía "Guerreros"

Director y Coreógrafo: Carlos Morúa Carrillo Bailarines: Santiago López y Alejandra Montero Fuente: Fotografía "Danzaton 2016", Teatro de la Danza corporaron seis estudiantes: Alexandra Delgado, Adrián Zumbado, Cristopher Aguilar, Antonio Granizo y Nelsyin Ruiz. Y a finales del año Josué Fonseca.

La última gira que realizó el grupo Humanizados en ese año, fue a San Carlos, gracias a la invitación de Oscar Chanis, donde participamos en el "I Festival de Coreógrafos San Carleño", se presentaron dos propuestas coreográficas en esta maravillosa actividad: "Metamorfosis" de Alejandra Montero, esta se estrenó en la segunda temporada de $\mathrm{Hu}$ manizados en el 2016 con el mismo nombre "Metamorfosis", la segunda coreografía que se presentó fue "Guerreros".

Ese fue un año increíble, aparte de graduarme como bachiller en Economía, inicié la licenciatura en esta carrera, el siguiente paso fue incorpórame al Colegio de Profesionales en Ciencias Económicas de Costa Rica (CPCECR), y como lo dice una canción muy famosa "gracias a la vida por haberme dado tanto" de Mercedes Sosa.

El CEG fue mi casa durante estos seis años, resumí lo que ha sido mi vida en 
el Grupo Humanizados, y en la UNA, no solo fui bailarín, coreógrafo y asistente en el grupo, también representé a la Universidad, en espectáculos, presentaciones en escuelas, colegios, comunidades y universidades en diferentes partes del país y en el extranjero; también me desempeñé como estudiante asistente, gracias al Lic. Enrique Mata (Exdecano del CEG) y a Dr. Miguel Baraona Cockere11 (Vicedecano del CEG), que me dieron la oportunidad, en la primera ocasión como asistente en la Cátedra de Humanismo para el Tercer Milenio (2014-2015), y en la segunda ocasión (2016), como asistente de la Dra. Maribel León en el Programa de Atención Integral de la Persona Adulta Mayor (PAIPAM), se me asignó el curso de "Emprendedores", donde compartí con un grupo de personas maravillosas, llenos de ideas y proyectos que deseaban desarrollar.

Quiero añadir que durante estos cinco años en el grupo Humanizados no recibí ningún reconocimiento o ayuda económica, mi labor fue exclusivamente por voluntad propia, el deseo de bailar y hacer la diferencia, que las personas entiendan que la danza está en todos y en todas partes, no hay que tener un título para bailar y menos para viajar por el mundo, solo un impulso que nos lleve a creer que se puede y que podemos transmitir el mensaje que queramos, se podrá tener la mejor técnica, pero las personas se conectan con las emociones, los sentimientos, algo que les permita creer, y que mueve los corazones; el verdadero baile para mí está en mover a la gente, que sientan algo.

Hubo un tiempo que estuve obsesionado con la técnica, deseaba mejorar por eso quería entrar a la Escuela de Danza, pues claro que la técnica es necesaria y esencial, pero lo que realmente quería era bailar, y eso es lo que he hecho durante este tiempo, bailar, no me arrepiento del rumbo que tomó mi vida, gracias a ello conocí muchas personas increíbles, profesionales humildes y he tenido una de las mejores experiencias de mi vida. 